\title{
Continuous-wave Semiconductor Disk Laser Emitting at 224 nm via Intracavity Frequency Tripling
}

\author{
Julio M. Rodríguez-García, David Pabœuf, Jennifer E. Hastie \\ Institute of Photonics, Department of Physics, SUPA, University of Strathclyde. The Technology and Innovation Centre, 99 George Street, \\ Glasgow G1 1RD, UK \\ julio.rodriguez@strath.ac.uk
}

\begin{abstract}
We present frequency tripling of a tunable continuous-wave red AlGaInP semiconductor disk laser. From a fundamental beam at $674 \mathrm{~nm}$, output power up to $\sim 100 \mu \mathrm{W}$ and laser tunability over $1.8 \mathrm{~nm}$ are reported.

OCIS codes: (140.7270) Vertical emitting lasers; (190.2620) Harmonic generation and mixing; (140.3610) Lasers, ultraviolet
\end{abstract}

\section{Introduction}

There is a lack of practical, continuous-wave laser sources in the UVC band (100 nm $-280 \mathrm{~nm})$, a potentially very useful region for several spectroscopic applications [1], enabling measurement of higher energy transitions of atmospheric species with high resolution. While there are already some UV laser sources, most require compromises. Few lasers combine high power, tunability, narrow linewidth, good beam quality and continuouswave operation. Semiconductor disk lasers (SDLs) have a quantum wells based gain medium that has been shown to enable compact and efficient laser sources in the visible and ultraviolet with extraordinary wavelength flexibility [2]. The surface emitting geometry satisfies the requirements for power scaling, while the broad gain bandwidth of the quantum wells enables tunability over tens of nanometres. The external cavity allows wavelength control of the laser with intracavity elements such as birefringent filters, while providing access to high intracavity powers, thus making SDLs suitable for taking advantage of several nonlinear effects.

Nonlinear frequency conversion has proven to be an efficient method to reach the ultraviolet region, either from near infrared or visible sources [3,4] or, more recently, using GaN laser diodes [5]. To date, intracavity second harmonic generation (SHG) has been shown to offer high power in both visible and UVA ranges of the electromagnetic spectrum. Similarly, sum-frequency generation has also proven to be an efficient process for the generation of UV radiation.

Here we demonstrate an intracavity frequency tripled SDL emitting at $224 \mathrm{~nm}$. This is the shortest wavelength emitted from an SDL to date and is the first implementation of intracavity frequency tripling in a visible SDL.

\section{Description of the laser source}

The experimental setup is described in Fig. 1. The SDL gain structure consists of ten pairs of 6-nm thick gallium indium phosphate (GaInP) quantum wells, separated by AlGaInP barriers. This gain medium is grown over a distributed Bragg reflector (DBR), composed of AlAs/AlGaAs layers. The whole structure is grown over a GaAs substrate.

The SDL is optically-pumped by a diode-pumped solid-state laser with up to $5.5 \mathrm{~W}$ of output power at $532 \mathrm{~nm}$. Thermal management is achieved by bonding a $500-\mu \mathrm{m}$-thick diamond heatspreader onto the intracavity surface of the SDL gain structure, clamped in a water-cooled brass mount (water temperature $2^{\circ} \mathrm{C}$ ). The pump beam is focused to a spot of $\sim 43 \mu \mathrm{m}$ radius which offers a high power density while avoiding thermal roll-over. The fundamental emission occurs at $\sim 674 \mathrm{~nm}$ so that deep UV emission at $\sim 224.5 \mathrm{~nm}$ can be achieved via tripling. In a first conversion step, intracavity second harmonic generation (SHG) in a lithium triborate (LBO) crystal is used to produce a UV beam at $\sim 337 \mathrm{~nm}$ (UVA), nearly collinear with the fundamental beam. LBO was chosen over other more efficient nonlinear crystals for the low spatial walk-off. The crystal is 7 -mm-long, cut for type I phase matching at $674 \mathrm{~nm}$ and $337 \mathrm{~nm}\left(\theta=90^{\circ}, \varphi=47.3^{\circ}\right)$. The fundamental beam and the UVA beam are then re-focused in a beta-barium borate (BBO) crystal, to produce the third harmonic via sum frequency mixing. This crystal is 5$\mathrm{mm}$-long and cut for type I phase matching at $674 \mathrm{~nm}, 337 \mathrm{~nm}$ and $224 \mathrm{~nm}\left(\theta=58.1^{\circ}\right)$. 
The laser setup (Fig. 1) consists of a 5-mirror cavity designed with 3 waists, at the SDL (43 $\mu$ m radius), the LBO crystal $(10 \mu \mathrm{m}$ radius $)$ and the BBO crystal ( $20 \mu \mathrm{m}$ radius) respectively. Tight waists have been chosen to ensure high power densities and thus favour higher conversion efficiencies. A 4-mm-thick birefringent filter (BRF) is used to narrow the linewidth and tune the wavelength. It also forces the polarisarion to be horizontal. The fundamental wavelength is centred around $674 \mathrm{~nm}$. SHG is achieved inside the LBO crystal, producing a laser beam at $337 \mathrm{~nm}$ propagating forward, nearly collinear with the fundamental beam. The faces of the crystal are anti-reflection coated for fundamental and second harmonic. The fourth mirror $\left(M_{4}\right.$ in Fig. 1) is high reflective for both wavelengths.

As only type I phase matching leads to reasonable non linear coefficient values for the SFG in BBO, it is necessary to rotate the polarisation of the second harmonic beam, so that it is parallel to that of the fundamental. A waveplate is inserted between the two crystals to this effect. The waveplate is designed to be half-wave at $337 \mathrm{~nm}$ and full-wave at $674 \mathrm{~nm}$. Both the fundamental and second harmonic beams are then focussed and overlapped inside the BBO crystal, setting up the conditions to produce third harmonic by sum-frequency generation. The face of the BBO next to the waveplate is anti-reflection coated for both the fundamental and the second harmonic beams, while the other face is anti-reflection coated for the fundamental and third harmonic beams. A beam at $224.5 \mathrm{~nm}$ is generated in the BBO crystal and is coupled out of the cavity by a dichroic mirror. This dichroic mirror is set at Brewster's angle and is high reflective for the third harmonic. Operation at Brewster's angle ensures high transmission for the fundamental beam. A prism is used outside of the cavity to separate the third harmonic from the remaining fundamental and second harmonic beams. Since the prism material is not fully transparent at $224 \mathrm{~nm}$ a large amount is absorbed. The transmission of the prism has been estimated as $50 \%$. All the power values later discussed are corrected accordingly to reflect the actual performance of the source.

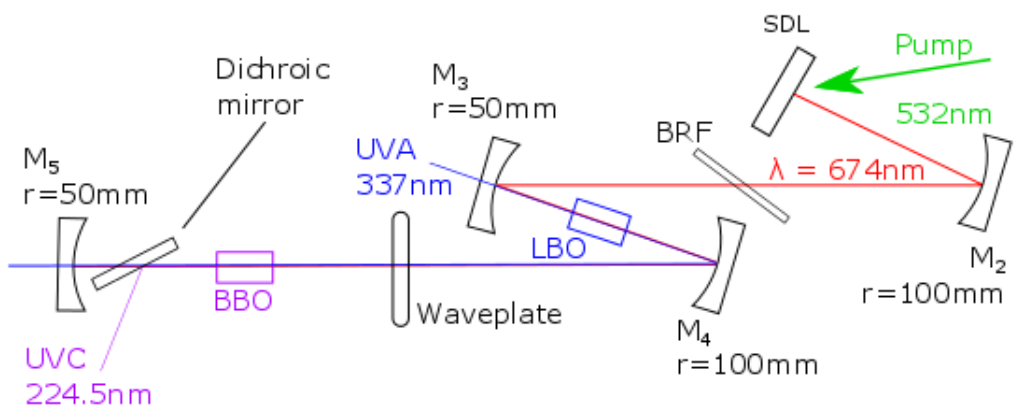

Fig. 1. Experimental setup. $M_{2-5}$ : HR cavity mirrors. BRF: birefringent filter.

\section{Laser characterizations}

Fig. 2a shows the emission spectrum of the laser with the third harmonic centred at $224.5 \mathrm{~nm}$. On this figure, one can also see the peak at $337 \mathrm{~nm}$ corresponding to the SH. The wavelength can be tuned between $223.8 \mathrm{~nm}$ and $225.5 \mathrm{~nm}$, by rotating the BRF and adjusting the phase matching angles of both crystals. Such a tuning range is quite remarkable $\left(\sim 360 \mathrm{~cm}^{-1}\right)$ and of potential interest for spectroscopy applications. The accessible wavelengths can potentially be extended by the use of different SDLs samples with emission centred around other wavelengths.

a)

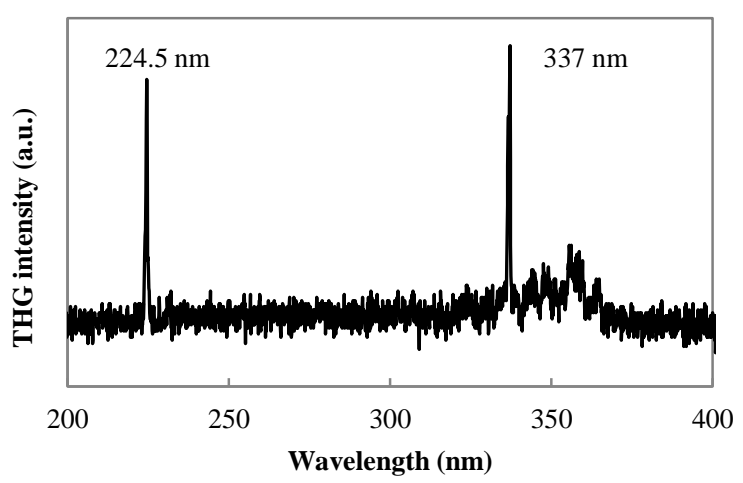

b)

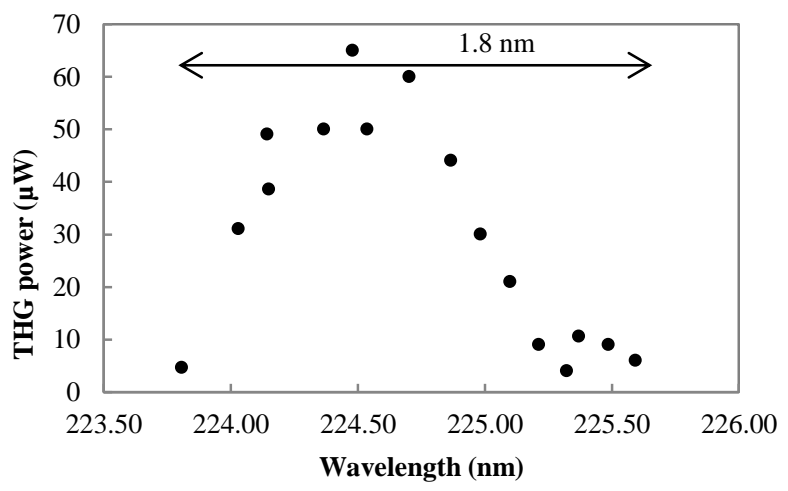

Fig. 2: a) Emission spectrum showing the second and the third harmonic. b) Tuning curve of the third harmonic. 
With $5.5 \mathrm{~W}$ of pump power incident on the SDL, around $100 \mu \mathrm{W}$ of third harmonic output are produced, as shown in Fig. 3. The intracavity power of the fundamental is estimated to be $26 \mathrm{~W}$, while the power of the second harmonic is $15 \mathrm{~mW}$. The threshold for the fundamental is around $1.35 \mathrm{~W}$ of pump power, but no THG can be detected below $2 \mathrm{~W}$. The efficiency of the second harmonic to third harmonic conversion is calculated to be $0.7 \%$, and we believe is limited by the overlap between the fundamental and the second harmonic beams. No evidence of thermal roll-over has been observed suggesting that higher output powers should be obtained with higher pump powers.

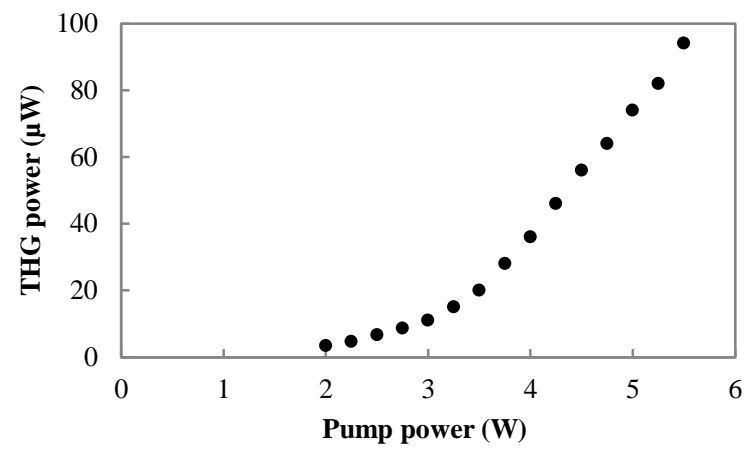

Fig. 3: Power transfer for the third harmonic.

A theoretical estimate of the THG power that should be achieved can be made by using the following formula derived from [6]:

$$
I_{3 \omega}=I_{2 \omega} \frac{\omega_{3 \omega}}{\omega_{2 \omega}}[\sin (\kappa z)]^{2}
$$

where $\kappa$ is the coupling coefficient, a function of the intracavity power, the wavelengths and the nonlinear coefficient of the crystal, z the length of the crystal. This equation assumes that only one beam (the SHG one in our case) is depleted by the sum frequency mixing. It also assumes perfect overlap between the beams and neglects walk off effects. With the current characteristics of the setup, these theoretical estimations would give a maximum power of $500 \mu \mathrm{W}$. We believe that the main limitation in our setup lays in the overlap between the fundamental and SHG during the sum-frequency process.

\section{Conclusion}

In summary, we have demonstrated here the shortest wavelength emitted from an SDL to date via the implementation of intracavity frequency tripling in a visible emitting SDL, providing an all-solid-state, continuouswave, tunable, UVC laser source. Powers up to $\sim 100 \mu \mathrm{W}$ have been observed. The laser is tunable over almost $2 \mathrm{~nm}$, corresponding to $\sim 360 \mathrm{~cm}^{-1}$. Further optimization of the conversion process is intended.

\section{References}

[1] C. Schulz, V. Sick, J. Heinze, and W. Stricker, "Laser-induced-fluorescence detection of nitric oxide in high-pressure flames with A-X(0, 2) excitation.," Appl. Opt. 36, 3227-32 (1997).

[2] S. Calvez, J. E. Hastie, M. Guina, O. G. Okhotnikov, and M. D. Dawson, "Semiconductor disk lasers for the generation of visible and ultraviolet radiation," Laser Photonics Rev. 3, 407-434 (2009).

[3] G. Hollemann, E. Peik, and H. Walther, "Frequency-stabilized diode-pumped Nd:YAG laser at $946 \mathrm{~nm}$ with harmonics at 473 and $237 \mathrm{~nm} ., "$ Opt. Lett. 19, 192 (1994).

[4] J. E. Hastie, L. G. Morton, A. J. Kemp, M. D. Dawson, A. B. Krysa, and J. S. Roberts, "Tunable ultraviolet output from an intracavity frequency-doubled red vertical-external-cavity surface-emitting laser," Appl. Phys. Lett. 89, 061114 (2006).

[5] N. Ruhnke, A. Müller, B. Eppich, R. Güther, M. Maiwald, B. Sumpf, G. Erbert, and G. Tränkle, "Single-pass UV generation at 222 . $5 \mathrm{~nm}$ based on high-power GaN external cavity diode laser," 2127-2129 (2015).

[6] Robert W. Boyd, Nonlinear Optics, Chapter 2, 3rd ed. (Academic Press, 2008). 